\title{
Effect of Dust Storms on FSO Communications
}

\section{Links}

\author{
Maged Abdullah Esmail ${ }^{\# 1}$, Habib Fathallah ${ }^{\# 2}$, and Mohamed-Slim Alouini ${ }^{* 3}$ \\ ${ }^{\#}$ Electrical Engineering Department, King Saud University \\ ${ }^{*}$ KACST Technology Innovation Center in Radio Frequency and Photonics (RFTONICs) \\ Riyadh 11421, Saudi Arabia \\ ${ }^{1}$ mesmailaksu.edu.sa.edu \\ ${ }^{2}$ hfathallaheksu.edu.sa \\ ${ }^{*}$ Computer, Electrical, and Mathematical Science and Engineering (CEMSE) Division, King Abdullah University \\ of Science and Technology (KAUST) \\ Thuwal, Makkah Province, Saudi Arabia \\ ${ }^{3}$ slim.alouini@kaust.edu.sa
}

\begin{abstract}
In literature, there is a lake of information about free space optic (FSO) systems' performance in arid and semi-arid areas that are prone to frequent dust storms. Therefore, in this paper, we investigate the performance of FSO links under dust storm conditions. We aim to determine the limits and capabilities of such systems in this harsh environment. To achieve this goal, we use some performance metrics including signal-to-noise (SNR) ratio, bit error rate (BER), and channel capacity. The results show that dust is a rough impairment that causes link drop under low visibility range. Moreover, we found that the system performance can be improved by using short segments or multi-hop system. Furthermore, the results show negligible improvement in system performance under dense dust. The comparison of fog and dust impairments show that dust introduces much higher attenuation than fog. Therefore, dust can be considered as the ultimate impairment for FSO links.
\end{abstract}

Keywords — FSO, next generation wireless networks, channel capacity, backhaul

\section{INTRODUCTION}

Advances in free space optic (FSO) technology in the last years have improved the FSO capability. FSO can be used as a solution for many applications including current and next generation of wireless networks, satellite communication, indoor communication, and under water communication etc. Current generation of wireless networks such as $4 \mathrm{G}$ promises $100 \mathrm{Mbps}$ download speed per user. In next generation 5G, higher data rate is required that may reach 10 Gbps [1]. However, current backhaul solutions such as DSL cables, microwave, etc. are not able to support this high speed. In contrast to these solutions, FSO is promising ultrahigh data rates and ultra-broadband capabilities. Current commercial FSO solutions for backhaul wireless networks support up to 10 Gbps speed which is considered as the highest among others [2]. Furthermore, lab demonstrations show much higher speed in the order of tens of Terabits per second $[3,4]$.

Although the capability of FSO to deliver high speed communication, there are some challenges that affect its performance. The primary challenge in outdoor FSO systems is weather condition represented by signal attenuation [5]. This attenuation ranges from less than one $\mathrm{dB} / \mathrm{km}$ in clear weather to hundreds of $\mathrm{dBs} / \mathrm{km}$ in harsh weather. Dust and fog are primary conditions that may reduce the visibility range to few meters and cause link outage. This is due to the comparable size of the dust particle with light wavelength used in FSO systems. Other conditions that have lower impact include snow, rain, haze, and scintillation.

The literature and information about dust effect on FSO is scarce. Actually, there is very little on the effect of dust and sand storms on FSO link. Because dust storm events are unexpected and unrepeatable, the authors in [6-8] build special experimental chambers to study the effect of dust storm on FSO links. In our recent work [8], we proposed a model for signal attenuation in dust using indoor chamber room. To the best of our knowledge, this is the first model to characterize the FSO signal attenuation under dust storm. This model is based on visibility range as an indication of the channel severity.

In this paper, we extend our work in [8] to investigate the FSO communication links under dust storm conditions using the proposed model. We demonstrate the limits and the requirements of establishing an FSO communication link in dust storm environment. We use some metrics to study the system performance including the bit error rate (BER), signalto-noise ratio (SNR), and channel capacity as a function of the visibility range. Moreover, power control and maximum reachable distance are discussed.

The remaining of the paper is organized as follows. In Section II, we discuss the dust storm characteristics and signal attenuation. In Section III, we study the FSO system model and study some performance metrics. The obtained results are discussed in Section VI. Finally, we conclude in Section V.

\section{DUST STORM CHARACTERISTICS AND FSO SIGNAL ATTENUATION}

Dust storms are hard weather conditions. They are 
TABLE I

DUST STORM CLASSIFICATION BASED ON VISIBILITY RANGE

\begin{tabular}{lcccc}
\hline \hline Dust Type & $\begin{array}{c}\text { Severe } \\
\text { dust storm }\end{array}$ & $\begin{array}{c}\text { Dust } \\
\text { storm }\end{array}$ & $\begin{array}{c}\text { Blowing } \\
\text { dust }\end{array}$ & $\begin{array}{c}\text { Dust } \\
\text { haze }\end{array}$ \\
\hline \hline Visibility $(\mathrm{km})$ & $<0.2$ & $0.2-1$ & $1-10$ & $\leq 10$ \\
\hline Description & Dense & Moderate & Light & Light \\
\hline \hline
\end{tabular}

characterized by strong winds and dust-filled air over wide area. Dust storm has particles size less than $100 \mu \mathrm{m}$. The dust storm causes strong turbulence winds and reduces visibility range to few meters. The storm can last from some minutes to several days.

Dust storms are divided into four classes according to the visibility range as listed in Table I [9]. The visibility range $(V)$ is defined as the distance over which the transmission drop to $2 \%$ of its initial value. Severe dust storm is the one with visibility range less than $200 \mathrm{~m}$. Second type is dust storm with higher visibility range from $200 \mathrm{~m}$ up to $1 \mathrm{~km}$. Light dust with visibility range from $1 \mathrm{~km}$ up to $10 \mathrm{~km}$ can be classified as blowing dust or haze. Blowing dust is due to dust blowing by wind while haze is due to dust storm that happens a distance away from the observation location.

In [8], we proposed a model for FSO signal attenuation in arid and semi-arid areas that are prone to frequent dust storms. The developed model is given by

$$
\alpha=52 \times V^{-1.05},(\mathrm{~dB} / \mathrm{km})
$$

where $V$ is the visibility in $\mathrm{km}$. This model is valid for $1550 \mathrm{~nm}$ wavelength which is the most common wavelength in FSO systems because it introduces lower absorption loss. Also its technology in terms of lasers and receivers is mature.

In next section, we will use this model to study, and evaluate the FSO system in terms of SNR, BER, and channel capacity. The obtained results will be used to determine the requirements and limits of this technology.
(IM/DD) FSO system with on-off keying (OOK) signaling which is widely used in research and commercial FSO products. In the work, we consider the effect of signal attenuation due to dust storm and geometrical losses. The optical received power signal at the receiver side is given by [10]

$$
P_{r}=P_{t}\left(D_{2} /\left(D_{1}+\theta_{t} L\right)\right)^{2} \tau_{t} 10^{(-\alpha L / 10)} \tau_{r},
$$

where $P_{t}$ is the transmitted power, $D_{1}$ and $D_{2}$ are the transmitter and receiver aperture diameter, $\theta_{\mathrm{t}}$ is the full transmitting divergence angle, $L$ is the link length, $\tau_{\mathrm{t}}$ is the transmitter optical efficiency, $\tau_{\mathrm{r}}$ is the receiver optical efficiency, and $\alpha$ is the atmospheric attenuation factor in $\mathrm{dB} / \mathrm{km}$.

In the receiver side, an avalanche photodiode (APD) is used which converts the received power signal into electrical current that is given by

$$
I_{P}=R P_{r},
$$

where $R$ represents the responsivity of the photodetector in $\mathrm{A} / \mathrm{W}$. The APD photodetector's SNR is given by [11]

$$
\gamma=\frac{M^{2} I_{p}^{2}}{2 e B\left(I_{p}+I_{D}\right) M^{(2+x)}+4 K T B F_{n} / R_{L}},
$$

where $M$ is the mean avalanche multiplication factor and $M^{\mathrm{x}}$ is the excess noise factor, $I_{D}$ is the dark current, $e$ is the electron charge, $B$ is the post-detection electrical bandwidth, $T$ is the absolute temperature, $K$ is the Boltzmann's constant, $R_{L}$ is the load resistor, and $F_{n}$ is the noise figure.

For NRZ-OOK signaling with NRZ, the BER of the system is given by [11]

$$
B E R=Q\left(\frac{\sqrt{\gamma}}{2}\right),
$$

where $Q$ is the Q-function. Another important metric to study the system performance is channel capacity. For OOK signaling, the lower bound channel capacity is asymptotically given by [12]

\begin{tabular}{|c|c|c|c|c|}
\hline & Parameter & value & parameter & value \\
\hline \multirow{2}{*}{ Transmitter } & Wavelength $(\lambda)$ & $1550 \mathrm{~nm}$ & Transmitter aperture diameter & $8 \mathrm{~cm}$ \\
\hline & Optical efficiency of transmitter $\tau_{\mathrm{t}}$ & 0.75 & Laser beam divergence angle $\theta$ & $2 \times 10^{-3} \mathrm{rad}$ \\
\hline \multirow{7}{*}{ Receiver } & Optical efficiency of receiver $\tau_{\mathrm{t}}$ & 0.75 & Responsivity $(R)$ & $0.7 \mathrm{~A} / \mathrm{W}$ \\
\hline & Receiver aperture diameter & $20 \mathrm{~cm}$ & Electrical bandwidth $(B)$ & $2.1 \mathrm{GHz}$ \\
\hline & Electron charge $(e)$ & $1.6 \times 10^{-19} \mathrm{C}$ & Noise figure $\left(F_{\mathrm{n}}\right)$ & 1 \\
\hline & Load resistance $\left(R_{\mathrm{L}}\right)$ & $1 \mathrm{k} \Omega$ & Avalanche multiplication factor $(M)$ & 40 \\
\hline & Boltzmann constant $(K)$ & $1.38 \times 10^{-23} \mathrm{~J} . \mathrm{k}$ & Excess avalance noise factor $\left(M^{\mathrm{x}}\right)$ & 400.7 \\
\hline & Temperature $(T)$ & $298 \mathrm{~K}$ & Data rate $(R)$ & $2.5 \mathrm{Gbps}$ \\
\hline & APD Dark current $\left(I_{\mathrm{D}}\right)$ & $10 \mathrm{nA}$ & Receiver sensitivity $\left(P_{\mathrm{s}}\right)$ & $\begin{array}{l}-34 \mathrm{dBm} @ \\
\mathrm{BER}=10-10\end{array}$ \\
\hline
\end{tabular}

\section{FSO CHANNEL MODEL}

We consider an intensity modulation/direct detection

TABLE I

PARAMETERS USED IN THE SIMULATION 


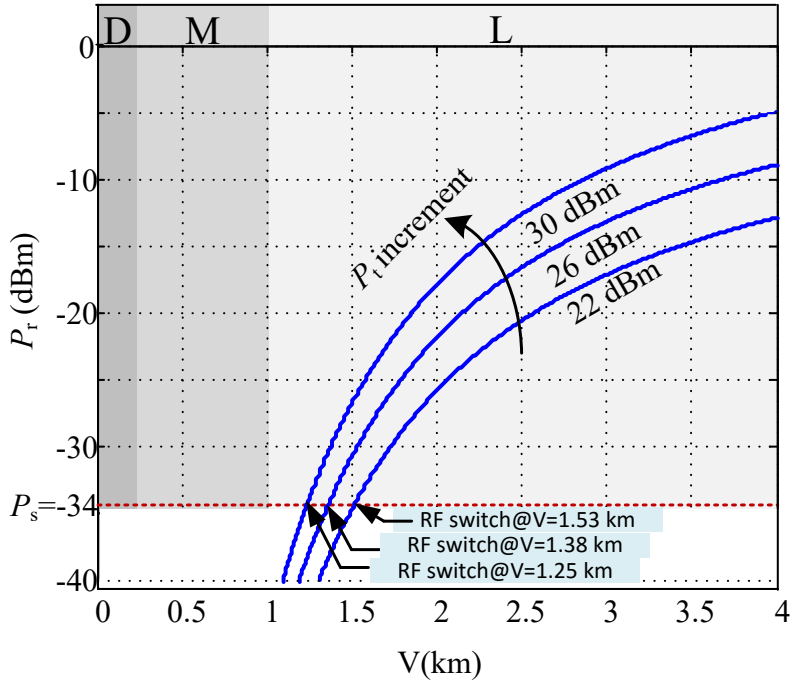

(a)

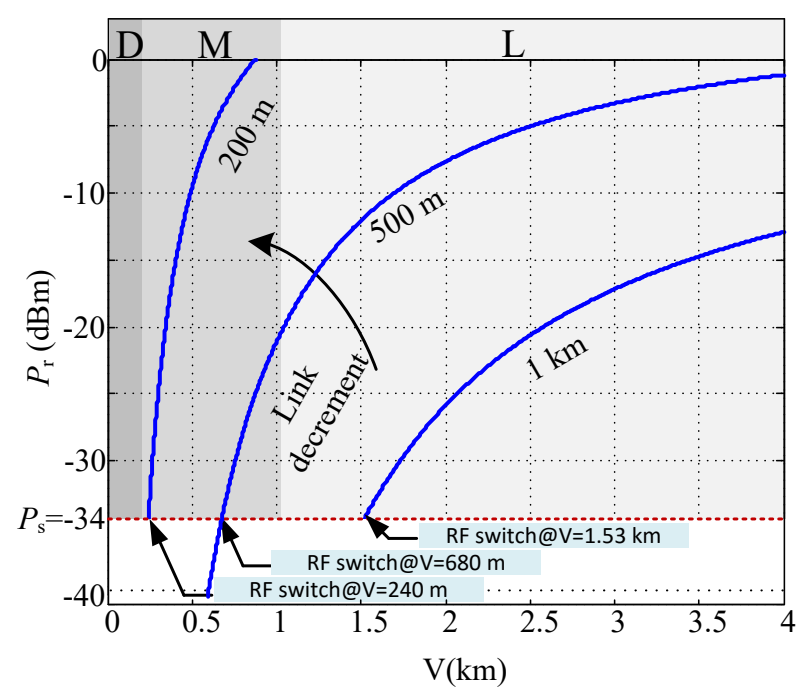

(b)

Fig. 1. Received power as a function of (a) the transmitted optical power, (b) link length with $P_{\mathrm{t}}=22 \mathrm{dBm}$. For received power below $P_{\mathrm{s}}=-34 \mathrm{dBm}$, we use RF backup link as illustrated in the figure.

$$
C \geq \frac{1}{2} \log _{2}\left(1+\frac{\mathrm{e} \gamma^{2}}{2 \pi}\right) \cdot \mathrm{bit} / \mathrm{sec} / \mathrm{Hz}
$$

\section{RESULTS AND DISCUSSIONS}

Using the performance metrics (2-6), in this section, we evaluate the FSO system performance under dusty conditions. In our simulation, we use the performance parameters and their associated values that are listed in Table II [8]. We divide the visibility range into four regions as mentioned before where $\mathrm{D}, \mathrm{M}$, and L stands for dense, moderate and light dust, respectively.

\section{A. Received power and link length}

The effect of the dusty channel on the system performance is shown in Fig. 1(a). The receiver sensitivity (threshold) is

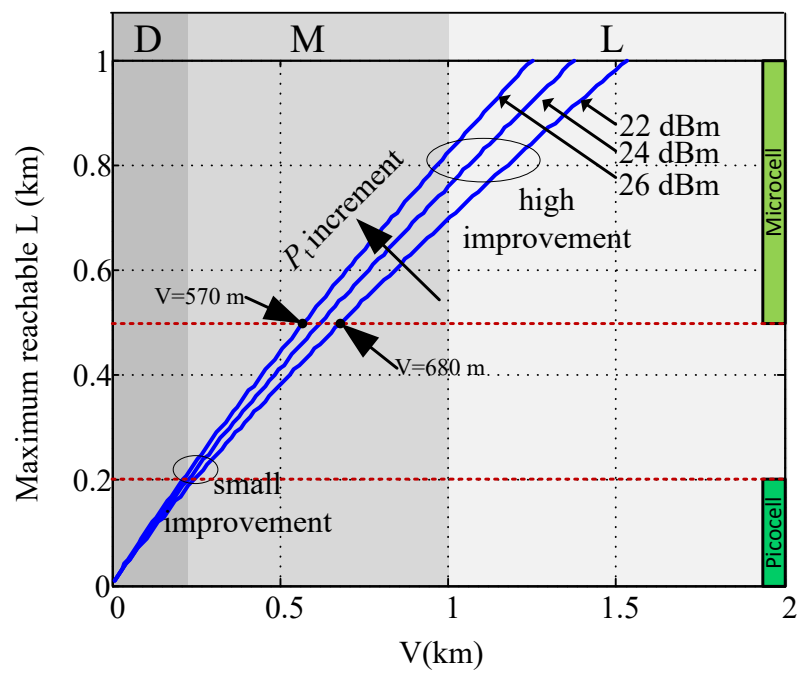

(a) assumed to be $-34 \mathrm{dBm}$. Once the received power drops below this threshold, the link drops. The results show that the system can work under light dust only, i.e. $V>1 \mathrm{~km}$. Under moderate and dense dust, the received power is less than the threshold. The curves show low improvement as we increase the transmitted power for low visibility values. In order to improve the system performance, RF backup link is needed. $\mathrm{FSO} / \mathrm{RF}$ systems have been proposed widely in literature and industry as a high reliability connection system [13]. Once the received power drops below this threshold, we use the low speed RF link to keep the connection till the dust severity reduces. For example, the system needs to switch to RF link when the visibility reduces to $1.25 \mathrm{~km}$ (respectively $1.38 \mathrm{~km}$ and $1.53 \mathrm{~km}$ ) for $22 \mathrm{dBm}$ (respectively $26 \mathrm{dBm}$ and $30 \mathrm{dBm}$ ) transmitted power.

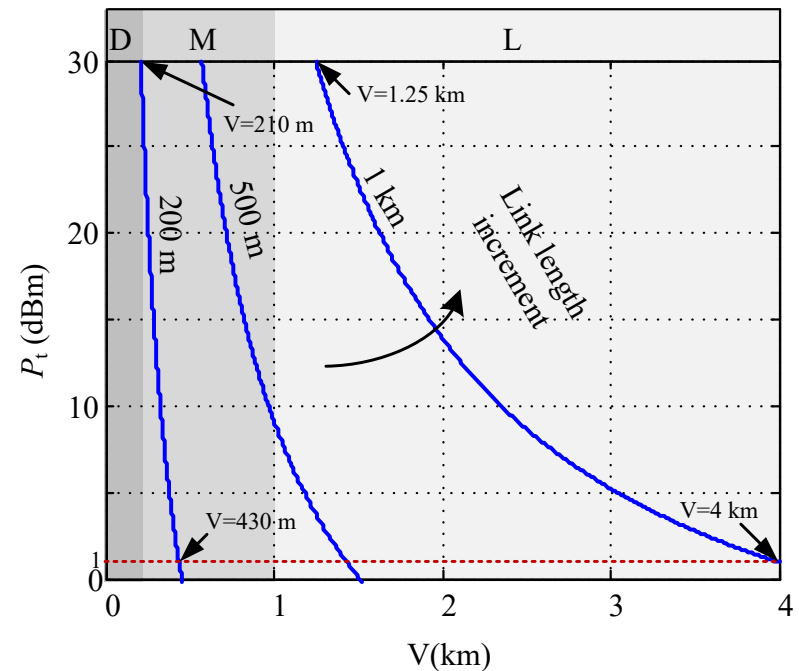

(b)

Fig. 2. Performance of FSO system showing (a) the maximum reachable link length, and (b) transmitted power control effect on the FSO system. We consider $P_{\mathrm{s}}=-34 \mathrm{dBm}$. 
Another way that helps reducing the effect of dust storm so that we can work under moderate and even dense dust is the use of multi-hop FSO links [14]. An erbium doped fiber amplification (EDFA) is used to amplify the signal and forward it to the next segment of the link. Using multiple segments instead of one single link improves the power budget and hence reduces the signal attenuation effect. The curves in this Fig. 1(b) shows high improvement in the system performance depending on the length of the link. For $1 \mathrm{~km}$ link length, the system is able to work under light dust only with $V>1.53 \mathrm{~km}$. Using $500 \mathrm{~m}$ (respectively $200 \mathrm{~m}$ ) link length, the system can work under moderate dust with $V>680 \mathrm{~m}$ (respectively $240 \mathrm{~m}$ ). However, under dense dust, shorter link less than $200 \mathrm{~m}$ is required to maintain a connection.

\section{B. Maximum reachable distance}

To determine how far an FSO system can work under dust conditions, we plot the maximum reachable range versus the visibility range in Fig. 2(a), assuming $P_{\mathrm{s}}=-34 \mathrm{dBm}$. As we notice, the distance increases as we increase the transmitted power under moderate and light dust conditions. However, under dense dust, there is no notable improvement in the maximum reachable distance. Under dense dust, the maximum possible distance is less than $200 \mathrm{~m}$. This distance increases under moderate dust to reach $800 \mathrm{~m}$. For light dust, the distance can be longer than $1 \mathrm{~km}$.

\section{Cellular cell size and link length}

Our discussion so far shows that FSO is a short range technology because of the outdoor limitations, mainly dust and fog. The current direction in cellular cells is to decease the cell size especially with proposing $\mathrm{mmW}$ technology for $5 \mathrm{G}$ wireless networks. In $5 \mathrm{G}$ networks, the cell size ranges from tens of meters to hundreds of meters. Hence, FSO can be a candidate as a backhaul solution to connect these cells because of its high capacity as compared to other technologies [15]. Fig. 2(a) shows also the effect of dust on the reach distance of wireless cells. For picocells that have size of few meters up to $200 \mathrm{~m}$, the FSO system can work under moderate and light dust with $200 \mathrm{~m}$ cell size. For dense dust, the cell size reduces. For example, if the visibility reduces to $100 \mathrm{~m}$, the cell size should be $100 \mathrm{~m}$ to keep connection. For microcells that have $500 \mathrm{~m}$ up to $2 \mathrm{~km}$ size, FSO can work under light dust and moderate dust with visibility larger than $680 \mathrm{~m}$ for $500 \mathrm{~m}$ link length. For example, if the visibility reduces below $680 \mathrm{~m}$ for $500 \mathrm{~m}$ cell size, power control can be used to compensate this loss. Moreover, in case the visibility reduces from $680 \mathrm{~m}$ to $570 \mathrm{~m}$, the link requires $8 \mathrm{~dB}$ more transmitted power.

\section{Transmitted power control}

The effect of controlling the transmitted power on the FSO system performance is shown in Fig. 2(b). We can see that under light dust, the system shows high improvement as we increase the transmitted power. However, this improvement decreases as we move to moderate dust and vanishes when we work under dense dust. For example, if we increase the transmitted power from $1 \mathrm{dBm}$ to $30 \mathrm{dBm}$ (29 dBm increase) under light dust with $1 \mathrm{~km}$ link length, the visibility range changes from $4 \mathrm{~km}$ to $1.25 \mathrm{~km}(2.75 \mathrm{~km}$ difference). For the same power increment ( $29 \mathrm{~dB}$ increase), only $220 \mathrm{~m}$ visibility range is achieved under dense dust with $200 \mathrm{~m}$ link length. Therefore, power controlling does not help to improve the system performance under dense dust. Note that the transmitted power cannot be increased unrestrictedly due to eye safety regulations.

\section{E. Signal-to-noise ratio (SNR), bit error rate (BER), and channel capacity}

Using (4), a plot of SNR under different link length and using different transmitted power values is shown in Fig. 3(a). The limit SNRs=14.8 $\mathrm{dB}$ corresponds to the receiver threshold. For $L=1 \mathrm{~km}$, the system can work under light dust

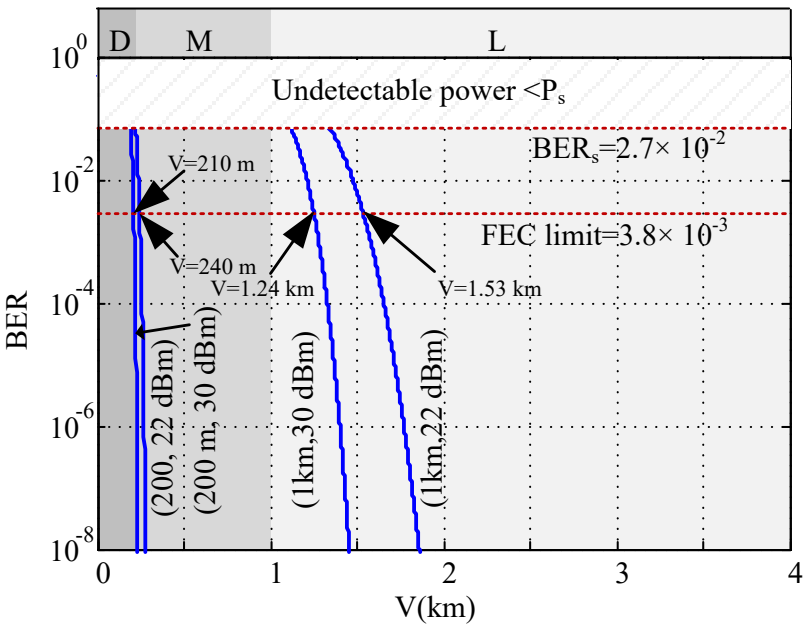

(b)

Fig. 3(a) SNR versus visibility as a function of power $\left(L, P_{\mathrm{t}}\right)$, (b) Bit error rate versus visibility as a function of power $\left(L, P_{\mathrm{t}}\right)$. 


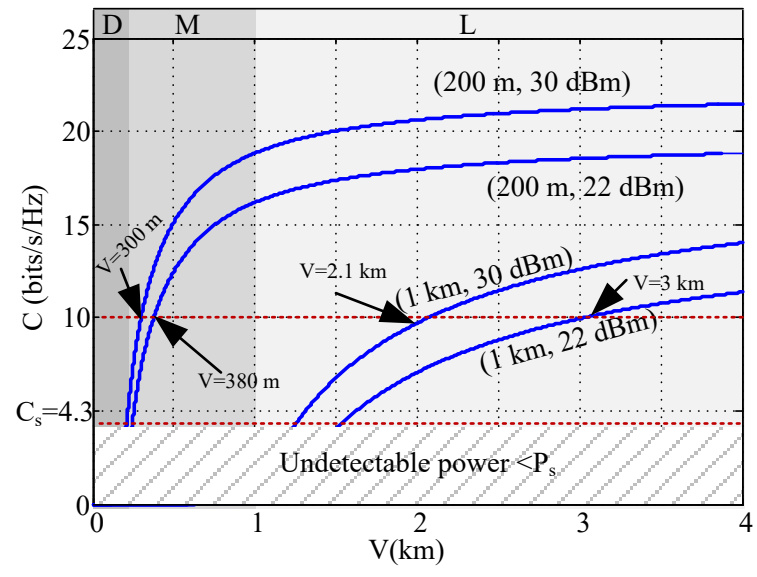

(a)

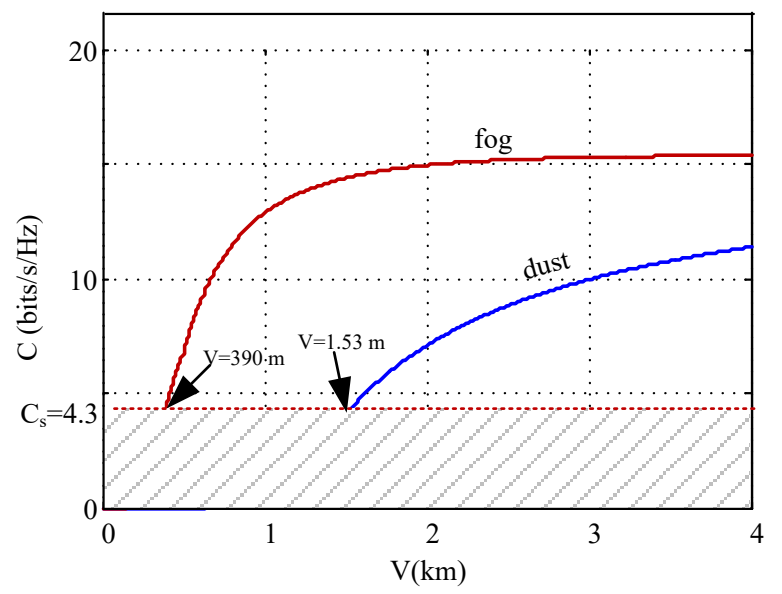

(b)

Fig. 4(a) channel capacity, versus visibility as a function of $\left(L, P_{\mathrm{t}}\right)$, (b) comparison between dust and fog signal in terms of channel capacity with $1 \mathrm{~km}$ link length and $P_{\mathrm{t}}=22 \mathrm{dBm}$.

only even with increasing the transmitted power. Decreasing the link length to $200 \mathrm{~m}$ improves highly the system performance allowing it to work under moderate dust conditions. However, under dense dust, the SNR drops below the limit. In that case, RF backup link is necessary to maintain the connection with the associated visibility range shown in Fig. 3(a).

Similar to SNR analysis, we used (5) to obtain the BER curves shown in Fig. 3(b) with $\mathrm{BERs}=2.7 \times 10^{-3}$ limit that corresponds to the receiver threshold. We notice a high degradation in system performance with low visibility. For $200 \mathrm{~m}$ link length, the achieved BER drops below the limit. So shorter link lengths are necessary to keep FSO system working. Such distance can be found in picocells that have size of few meters up to $200 \mathrm{~m}$. For BERs $=3.8 \times 10^{-3}$ which is considered as error free limit because errors can be corrected by forward error correction (FEC) codes, we achieve $210 \mathrm{~m}$, $240 \mathrm{~m}, 1.24 \mathrm{~km}$, and $1.53 \mathrm{~km}$ visibility range for the following link length and transmitted power: $(200 \mathrm{~m}, 30 \mathrm{dBm}),(200 \mathrm{~m}$, $22 \mathrm{dBm}),(1 \mathrm{~km}, 30 \mathrm{dBm})$, and $(1 \mathrm{~km}, 22 \mathrm{dBm})$, respectively.

Finally, we show the performance of FSO system in terms of channel capacity in Fig. 4(a). The capacity limit that corresponds to the receiver sensitivity is $C_{\mathrm{s}}=4.3 \mathrm{~b} / \mathrm{s} / \mathrm{Hz}$. Similar to SNR and BER, the system cannot support links with a length larger than $200 \mathrm{~m}$ under dense dust. Shorter links show better performance than increasing the transmitted power. For $C=10 \mathrm{~b} / \mathrm{s} / \mathrm{Hz}$ and $200 \mathrm{~m}$ link length, we achieve $300 \mathrm{~m}$ and $380 \mathrm{~m}$ visibility range with $30 \mathrm{dBm}$ and $22 \mathrm{dBm}$, respectively. For $1 \mathrm{~km}$ link length, we achieved the same capacity at $2.1 \mathrm{~km}$ and $3 \mathrm{~km}$ visibility range with $30 \mathrm{dBm}$ and $22 \mathrm{dBm}$, respectively.

\section{F. Comparing the effect of dust and fog}

So far we discussed the effect of dust on the FSO communication links. Most of the literature work in FSO channel modeling considers fog as the harshest environment for FSO systems. This is because such work was performed in foggy environment in Europe and North America [5]. In this work, we compare the effect of fog and dust on the FSO links and determine which one has more effect. For fog conditions, there are some reported models in literature. We will use Kim model which is a key channel model to determine the signal attenuation. This model depends on the visibility range which is given by [5]

$$
A=\frac{13}{V}\left(\frac{\lambda}{550}\right)^{-q},(\mathrm{~dB} / \mathrm{km})
$$

where $V$ is the visibility range in $\mathrm{km}, \lambda$ is the signal wavelength in $\mu \mathrm{m}$, and the coefficient $q$ is given by

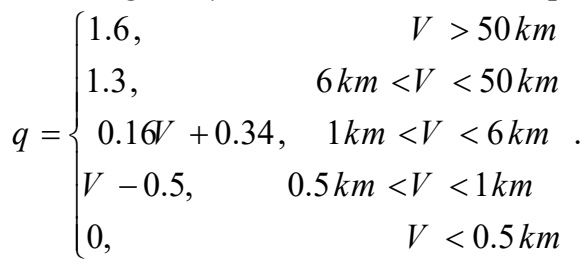

Fig. 4(b) shows the channel capacity for FSO system under dust and fog attenuation. The results show better performance under fog conditions. For example, at $4.3 \mathrm{~b} / \mathrm{s} / \mathrm{Hz}$ capacity, the FSO system works at $1.53 \mathrm{~km}$ visibility range under dust while under fog this range reduces to $390 \mathrm{~m}$. Therefore, the effect of dust is harder than fog on the FSO links.

\section{CONCLUSIONS}

Most of the work in literature ignores the dust storm effect of FSO communication links because the carried research was performed in areas lacking such environment. In this paper, we investigated the effect of dust storm based on recent empirical model proposed to predict the signal attenuation. Our results show that dust storm has high impact on the system performance especially under dense dust where the possibility of maintaining connection vanishes. According to this study, FSO could have a main role in next generation of wireless networks that have size in hundreds of meters.

\section{ACKNOWLEDGMENT}

This work was supported by King Abdulaziz City for Science and Technology (KACST) under Project APR 34-145. 


\section{REFERENCES}

[1] E. Hossain, M. Rasti, H. Tabassum, and A. Abdelnasser, "Evolution toward 5G multi-tier cellular wireless networks: An interference management perspective," IEEE Wir.Comm., vol. 21, pp. 118-127, 2014.

[2] Artolink. $10 \mathrm{Gbit} / \mathrm{s}$ Artolink model. Available: http://artolink.com/page /products/free_space_optics_Artolink_10Gbps/

[3] H. Huang, et al. , "100 Tbit/s free-space data link enabled by threedimensional multiplexing of orbital angular momentum, polarization, and wavelength," Optics letters, vol. 39, pp. 197-200, 2014.

[4] M. A. Esmail, A. Ragheb, H. Fathallah, and M. S. Alouini, "Experimental demonstration of outdoor 2.2 Tbps super-channel FSO transmission system," in 2016 IEEE International Conference on Communications Workshops (ICC), 2016, pp. 169-174.

[5] M. A. Esmail, H. Fathallah, and M. S. Alouini, "Outdoor FSO Communications under Fog: Attenuation Modeling and Performance Evaluation," IEEE Photonics Journal, vol. 8, pp. 1-22, 2016.

[6] K. Su, L. Moeller, R. B. Barat, and J. F. Federici, "Experimental comparison of terahertz and infrared data signal attenuation in dust clouds," JOSA A, vol. 29, pp. 2360-2366, 2012.

[7] Z. Ghassemlooy, J. Perez, and E. Leitgeb, "On the performance of FSO communications links under sandstorm conditions," in 12th International Conference on Telecommunications, 2013, pp. 53-58.

[8] M. Esmail, H. Fathallah, and M.-S. Alouini, "An Experimental Study of FSO Link Performance in Desert Environment," IEEE Communications Letters, vol. PP, no. 99, pp.1-4, 2016.

[9] Y. Shao, Physics and modelling of wind erosion vol. 37: Springer, 2008.

[10] A. K. Majumdar and J. C. Ricklin, Free-space laser communications: principles and advances vol. 2: Springer, 2010.

[11] J. M. Senior and M. Y. Jamro, Optical fiber communications: principles and practice: Pearson Education, 2009.

[12] A. Chaaban, J.-M. Morvan, and M.-S. Alouini, "Free-space optical communications: Capacity bounds, approximations, and a new spherepacking perspective," IEEE Transactions on Communications, vol. 64, pp. 1176-1191, 2016.

[13] M. Usman, H.-C. Yang, and M.-S. Alouini, "Practical switching-based hybrid $\mathrm{FSO} / \mathrm{RF}$ transmission and its performance analysis," IEEE Photonics Journal, vol. 6, pp. 1-13, 2014.

[14] E. Bayaki, D. S. Michalopoulos, and R. Schober, "EDFA-based alloptical relaying in free-space optical systems," IEEE Transactions on Communications, vol. 60, pp. 3797-3807, 2012.

[15] T. S. Rappaport, et al., "Millimeter wave mobile communications for 5G cellular: It will work!," IEEE access, vol. 1, pp. 335-349, 2013. 